\title{
COEFFICIENTS FOR THE AREA THEOREM
}

\author{
A. W. GOODMAN ${ }^{1}$
}

ABstract. Let $f(z)=\sum_{n=1}^{\infty} a_{n} z^{n}$, and set $G(z)=f\left(z^{-p}\right)^{-1 / p}=$ $\sum_{n=0}^{\infty} g_{n p-1} z^{1-n p}$. This paper finds an explicit formula for $g_{n p-1}$ in terms of the $a_{n}$. Such a formula (apparently previously unknown) may be very useful in the theory of univalent functions.

1. Introduction. The importance of the area theorem in the theory of univalent functions is well known [1]. One form of this theorem [8, p. 209] runs as follows.

THEOREM A. Suppose that $f(z)$ is regular and univalent in $|z|<1$, and

$$
f(z)=z+\sum_{n=2}^{\infty} a_{n} z^{n}
$$

Define $G(z)$ by

$$
G(z)=\frac{1}{f(1 / z)}=z+\sum_{n=0}^{\infty} \frac{g_{n}}{z^{n}}
$$

Then

$$
\sum_{n=1}^{\infty} n\left|g_{n}\right|^{2} \leqq 1
$$

Although the inequality (3) is useful in obtaining properties of the function $f(z)$ and domains for the coefficients $a_{n}$, this usefulness is somewhat limited because the $g_{n}$ are complicated functions of the $a_{n}$, and the computation of these functions is time consuming for large $n$ (here large means $n>4$ ). As far as the author is aware no general formula for $g_{n}$ has been given up to this time. The purpose of this paper is to present such a formula.

Although our methods are different from those used by Hummel [4], it was his excellent treatment of an analogous problem in determining the Grunsky coefficients that encouraged the author to search for the formula given below.

Presented to the Society, November 20, 1971; received by the editors May 27, 1971. AMS 1970 subject classifications. Primary 30A28, 30A34; Secondary 30A36.

Key words and phrases. Univalent functions, area theorem, coefficient bounds.

${ }^{1}$ This research was supported by National Science Foundation Grant GP-18558.

c. American Mathematical Society 1972 
It should be mentioned that the inequality (3) is quite often misnamed. A study of the papers involved [2], [3] clearly shows that (3) was first proved by Gronwall, and consequently we refer to it as Gronwall's inequality. This priority of Gronwall has also been noticed by Jenkins $[5$, p. 2].

2. The formula. It is often useful to consider $\left(f\left(z^{2}\right)\right)^{1 / 2}$ rather than $f(z)$. More generally we let $p$ be an arbitrary positive integer, and we replace (2) by

$$
G(z)=\left(f\left(1 / z^{p}\right)\right)^{-1 / p}=z+\sum_{n=1}^{\infty} \frac{g_{n p-1}^{(p)}}{z^{n p-1}} .
$$

Direct and laborious computations give the following formulas

(5) $\quad g_{p-1}^{(p)}=-\frac{1}{p} a_{2}$

(6) $g_{2 p-1}^{(p)}=-\frac{1}{p} a_{3}+\frac{p+1}{2 p^{2}} a_{2}^{2}$,

(7) $g_{3 p-1}^{(p)}=-\frac{1}{p} a_{4}+\frac{p+1}{p^{2}} a_{2} a_{3}-\frac{(2 p+1)(p+1)}{6 p^{3}} a_{2}^{3}$,

$$
\begin{aligned}
g_{4 p-1}^{(p)}= & -\frac{1}{p} a_{5}+\frac{p+1}{p^{2}} a_{2} a_{4}+\frac{p+1}{2 p^{2}} a_{3}^{2} \\
& -\frac{(2 p+1)(p+1)}{2 p^{3}} a_{2}^{2} a_{3}+\frac{(3 p+1)(2 p+1)(p+1)}{24 p^{4}} a_{2}^{4}
\end{aligned}
$$

Before proceeding, it is convenient to introduce a compact notation for the products that are beginning to appear in (7) and (8). Indeed we set

$$
\gamma(p, m)=(p+1)(2 p+1) \cdots(m p+1)=\prod_{j=1}^{m}(j p+1)
$$

with the usual convention that $\gamma(p, 0)=1$. Then

$$
\begin{aligned}
g_{5 p-1}^{(p)}= & -\frac{1}{p} a_{6}+\frac{p+1}{p^{2}} a_{2} a_{5}+\frac{p+1}{p^{2}} a_{3} a_{4} \\
& -\frac{\gamma(p, 2)}{2 p^{3}}\left(a_{2}^{2} a_{4}+a_{2} a_{3}^{2}\right)+\frac{\gamma(p, 3)}{6 p^{4}} a_{2}^{3} a_{4}-\frac{\gamma(p, 4)}{120 p^{5}} a_{2}^{5}
\end{aligned}
$$




$$
\begin{aligned}
g_{6 p-1}^{(p)}= & -\frac{1}{p} a_{7}+\frac{p+1}{p^{2}}\left(a_{2} a_{6}+a_{3} a_{5}+\frac{a_{4}^{2}}{2}\right) \\
& -\frac{\gamma(p, 2)}{p^{3}}\left(\frac{a_{2}^{2} a_{5}}{2}+a_{2} a_{3} a_{4}+\frac{a_{3}^{3}}{6}\right) \\
& +\frac{\gamma(p, 3)}{p^{4}}\left(\frac{a_{2}^{3} a_{4}}{6}+\frac{a_{2}^{2} a_{3}^{2}}{4}\right) \\
& -\frac{\gamma(p, 4)}{24 p^{5}} a_{2}^{4} a_{3}+\frac{\gamma(p, 5)}{720 p^{6}} a_{2}^{6} .
\end{aligned}
$$

We have listed the first six cases of our general formula explicitly because the sixth case, equation (11), is the first one that involves $a_{7}$, and 7 is the smallest integer for which the general conjecture, $\left|a_{n}\right| \leqq n$, is currently open.

The general formula is almost obvious from the six cases already cited. However to express it in a simple way it is convenient to set $b_{n}=a_{n+1}$, for $n=1,2, \cdots$, in order to bring to the surface a certain order in the formulas that might otherwise go unnoticed (see Hummel [4]).

THEOREM 1. Let $S(n)$ be the set of all $n$-tuples $\left(r_{1}, r_{2}, \cdots, r_{n}\right)$ of nonnegative integers for which

$$
r_{1}+2 r_{2}+3 r_{3}+\cdots+n r_{n}=n,
$$

and for each such $n$-tuple define $m$ by

$$
r_{1}+r_{2}+\cdots+r_{n}=m \text {. }
$$

If $b_{n}=a_{n+1}, n=1,2, \cdots$, and $g_{n p-1}^{(p)}$ is defined by equation (4) where $f(z)$ is given by (1), then

$$
\boldsymbol{g}_{n p-1}^{(p)}=\sum \frac{(-1)^{m} \gamma(p, m-1) b_{1}^{r_{1}} b_{2}^{r_{2}} \cdots b_{n}^{r_{n}}}{p^{m} r_{1} ! r_{2} ! \cdots r_{n} !}
$$

where the sum is over all $n$-tuples in $S(n)$.

It is a simple matter to check that the formula (14) gives (5), (6), (7), (8), (10) and (11) when $n=1,2,3,4,5$, and 6 respectively.

Proof. For simplicity we drop the superscript on $g$. We differentiate the identity (4) and then set $1 / z^{p}=\zeta$. After a few minor steps we find that

$$
\frac{\zeta f^{\prime}(\zeta)}{f(\zeta)} \frac{G(z)}{z}=\sum_{n=0}^{\infty}(1-n p) g_{n p-1} \zeta^{n}
$$


with $g_{-1} \equiv 1$. The power series for $f, f^{\prime}$, and $G$ transform (15) into

$$
\left(\sum_{n=1}^{\infty} n a_{n} \zeta^{n}\right)\left(\sum_{n=0}^{\infty} g_{n p-1} \zeta^{n}\right)=\left(\sum_{n=1}^{\infty} a_{n} \zeta^{n}\right)\left(\sum_{n=0}^{\infty}(1-n p) g_{n p-1} \zeta^{n}\right) .
$$

For fixed integer $n \geqq 1$ we equate coefficients of $\zeta^{n+1}$ in (16) and find

$$
\sum_{k=0}^{n}(n-k+k p) a_{n+1-k} g_{k p-1}=0
$$

Since $a_{1}=1$, we can solve (17) for $g_{n p-1}$. This gives

$$
g_{n p-1}=-\frac{1}{n p} \sum_{k=0}^{n-1}(n-k+k p) a_{n+1-k} g_{k p-1}
$$

Now, equation (18) is a recursion formula that allows us to compute $g_{n p-1}$ from the ones with smaller index, and as such determines the sequence of coefficients $g_{n p-1}$ in a unique manner. Consequently, in order to prove Theorem 1, it is sufficient to show that for each index $n$ the coefficients $g_{n p-1}$ defined by equation (14) do indeed satisfy (18). Again it is an easy matter to check that if we set $n=1,2,3,4,5$, and 6 in (18) this formula does give the first six coefficients aiready listed.

As already indicated we replace $a_{n+1-k}$ by $b_{n-k}$. Then (18) becomes

$$
g_{n p-1}=-\frac{1}{n p} \sum_{k=0}^{n-1}(n-k+k p) b_{n-k} g_{k p-1} \text {. }
$$

We proceed by induction. That is, we assume that for each $k=1,2, \cdots$, $n-1$,

$$
g_{k p-1}=\sum \frac{(-1)^{j} \gamma(p, j-1) b_{1}^{r_{1}} b_{2}^{r_{2}} \cdots b_{k}^{r_{k}}}{p^{j r_{1} ! r_{2} ! \cdots r_{k} !}}
$$

where $j \equiv r_{1}+r_{2}+\cdots+r_{k}$ and the sum is over $S(k)$ the set of all nonnegative $k$-tuples $\left(r_{1}, r_{2}, \cdots, r_{k}\right)$ for which $r_{1}+2 r_{2}+\cdots+k r_{k}=k$. Now if $k<n$, it does no harm to enlarge the $k$-tuple to an $n$-tuple by adjoining a suitable number of zeros. On the one hand any solution of

$$
r_{1}+2 r_{2}+\cdots+n r_{n}=k, \quad k<n,
$$

in nonnegative integers must give $r_{i}=0$, if $i=k+1, k+2, \cdots, n$. On the other hand the inclusion of the factors $b_{i}^{r_{i}} / r_{i}$ ! in (20) causes no harm because for $i=k+1, k+2, \cdots, n$ these factors are 1 . Consequently (20) can be replaced by

$$
\dot{g}_{k p-1}=\sum \frac{(-1)^{j} \gamma(p, j-1) b_{1}^{r_{1}} b_{2}^{r_{2}} \cdots b_{n}^{r_{n}}}{p^{j} r_{1} ! r_{2} ! \cdots r_{n} !}
$$


where $k \leqq n, j \equiv r_{1}+r_{2}+\cdots+r_{n}$, and the sum is over the set $S(k)$ of all nonnegative integer solutions of (21). We use (22) in the right side of (19). Then for the right side $R$, we have

$$
R \equiv-\frac{1}{n p} \sum_{k=0}^{n-1} \sum_{S(k)} \frac{(-1)^{j}(n-k+k p) \gamma(p, j-1) b_{n-k} b_{1}^{r_{1}} b_{2}^{r_{2}} \cdots b_{n}^{r_{n}}}{p^{j} r_{1} ! r_{2} ! \cdots r_{n} !}
$$

Now let $\left(s_{1}, s_{2}, \cdots, s_{n}\right)$ be any fixed $n$-tuple in $S(n)$, so that

$$
\sum_{i=1}^{n} i s_{i}=n, \quad \sum_{i=1}^{n} s_{i}=m
$$

We are to determine the coefficient $C$ of $b_{1}^{s_{1}} b_{2}^{s_{2}} \cdots b_{n}^{s_{n}}$ in (23). This coefficient may arise from combining several terms from the sum and in fact such terms arise if and only if $b_{n-k} b_{1}^{r_{1}} b_{2}^{r_{2}} \cdots b_{n}^{r_{n}}=b_{1}^{s_{1}} b_{2}^{s_{2}} \cdots b_{n}^{s_{n}}$. To be specific let $\alpha$ be an index for which $s_{\alpha} \geqq 1$, and let $r_{i}=s_{i}$ if $i \neq \alpha$, and let $r_{\alpha}=s_{\alpha}-1$. For this fixed $\alpha$, we have $j=\sum_{i=1}^{n} r_{i}=m-1$. In (23) we set $n-k=\alpha$. If $A$ is the set of $\alpha$ for which $s_{\alpha} \neq 0$, then

$$
C=-\frac{1}{n p} \sum_{\alpha \in A} \frac{(-1)^{m-1}(\alpha+(n-\alpha) p) \gamma(p, m-2)}{p^{m-1} r_{1} ! r_{2} ! \cdots r_{n} !} .
$$

Inserting the factor $s_{\alpha}$ in the numerator and denominator of (25) we have

$$
\begin{aligned}
C & =\sum_{\alpha \in A} \frac{(-1)^{m} s_{\alpha}(\alpha+(n-\alpha) p) \gamma(p, m-2)}{n p^{m} s_{1} ! s_{2} ! \cdots s_{n} !} \\
& =\frac{(-1)^{m} \gamma(p, m-2)}{n p^{m} s_{1} ! s_{2} ! \cdots s_{n} !} \sum_{\alpha \in A} s_{\alpha}(\alpha+(n-\alpha) p) .
\end{aligned}
$$

But if $s_{\alpha}=0$, the corresponding term in the sum is zero, hence (using (24))

$$
\begin{aligned}
C & =\frac{(-1)^{m} \gamma(p, m-2)}{n p^{m} s_{1} ! s_{2} ! \cdots s_{n} !} \sum_{\alpha=1}^{n}\left(\alpha s_{\alpha}+n p s_{\alpha}-p \alpha s_{\alpha}\right) \\
& =\frac{(-1)^{m} \gamma(p, m-2)}{n p^{m} s_{1} ! s_{2} ! \cdots s_{n} !}(n+n p m-p n) \\
& =\frac{(-1)^{m} \gamma(p, m-2)}{p^{m} s_{1} ! s_{2} ! \cdots s_{n} !}((m-1) p+1)=\frac{(-1)^{m} \gamma(p, m-1)}{p^{m} s_{1} ! s_{2} ! \cdots s_{n} !} .
\end{aligned}
$$

But this is precisely the coefficient of $b_{1}^{s_{1}} b_{2}^{s_{2}} \cdots b_{n}^{s_{n}}$ required in formula (14). since the argument holds for each fixed $\left(s_{1}, s_{2}, \cdots, s_{n}\right)$ this completes the proof of Theorem 1 . 
3. Remarks. Although the formula for $g_{n p-1}$ was derived for $p$ a positive integer, and it was assumed that $f(z)$ is univalent in $|z|<1$, the formula is independent of both of these assumptions. The only requirement on $f(z)$ is that $f(z)$ have a simple zero at $z=0$ and $f^{\prime}(0)=1$. The power series (4) for $G(z)$ wili converge for $i z \mid>R_{0}$ where $R_{0}=1 / r_{0}$ and $r_{0}$ is the modulus of the smallest zero of $f(z) / z$. Further $p$ may be any integer positive or negative, as long as $p \neq 0$. We can obtain numerical checks on the formula, and deduce various identities by selecting special values for $p$ and special functions. For example if $p=-1$, then $G(z) \equiv f(z)$. This explains the presence of the factor $p+1$ in every term except the term $-a_{n} / p$ in (14).

Suppose that $p=-2$. Then

$$
G(z)=\left(f\left(z^{2}\right)\right)^{1 / 2} \equiv z+\sum_{n=1}^{\infty} c_{2 n+1} z^{2 n+1},
$$

and (14) gives

$$
c_{2 n+1}=\frac{1}{2} a_{n+1}+\sum_{S(n) ; m>1} \frac{(-1)^{m+1}(2 m-3) ! a_{2}^{r_{1}} a_{3}^{r_{2}} \cdots a_{n+1}^{r_{n}}}{2^{2 m-2}(m-2) ! r_{1} ! r_{2} ! \cdots r_{n} !},
$$

a formula for the coefficients in the square root of a power series.

The case $p=1$ gives a formula for the reciprocal of a power series. Thus if

then for $n \geqq 1$,

$$
g(z)=\frac{1}{f(z)}=\frac{1}{z}+\sum_{n=0}^{\infty} d_{n} z^{n}
$$

$$
d_{n-1}=\sum_{S(n)} \frac{(-1)^{m} m ! a_{2}^{r_{1}} a_{3}^{r_{2}} \cdots a_{n+1}^{r_{n}}}{r_{1} ! r_{2} ! \cdots r_{n} !}
$$

It would be convenient to have a formula that would give the number of elements in the set $S(n)$. However, this is too much to expect because this number is $p(n)$, the number of unrestricted partitions of $n$. The function $p(n)$ has been the subject of intensive research since the days of Euler, and although much is known [6], [7], a simple formula for $p(n)$ has not been found and it is doubtful if such a formula exists. Many of the properties of $p(n)$ follow from the relation

$$
\frac{1}{(1-z)\left(1-z^{2}\right)\left(1-z^{3}\right)\left(1-z^{4}\right) \cdots}=1+\sum_{n=1}^{\infty} p(n) z^{n} .
$$

Values of $p(n)$ have been computed for $n \leqq 1000$, and may be folind in the Royal Society Mathematics Tables, vol. 4, Cambridge Univ. Press, London, 1958, pp. 118-121. 


\section{REFERENCES}

1. S. D. Bernardi, A bibliography of schlicht functions, Courant Institute of Mathematical Sciences, New York University, Tech. Report \#NR 041-019, IMM 351, Office of Naval Research, New York; New York University, New York, 1966. MR 34 \#2849.

2. L. Bieberbach, Uber die Koeffizienten derjenigen Potenzreihen, welche eine schlichte Abbildung des Einheitskreises vermitteln, S.-B. Preuss. Akad. Wiss. 38 (1916), 940-955.

3. T. H. Gronwall, Some remarks on conformal representation, Ann. of Math. (2) $16(1914 / 15), 72-76$.

4. J. A. Hummel, The Grunsky coefficients of a schlicht function, Proc. Amer. Math. Soc. 15 (1964), 142-150. MR 28 \#1287.

5. J. A. Jenkins, Univalent functions and conformal mappings, 2nd rev. ed., SpringerVerlag, Berlin, 1965. MR 20 \#3288.

6. P. A. MacMahon, Combinatory analysis. Vols. I, II, Cambridge Univ. Press, New York, 1915, 1916.

7. I. Niven and H. S. Zuckerman, An introduction to the theory of numbers, Wiley, New York, 1960. MR 22 \#5605.

8. E. C. Titchmarsh, The theory of functions, 2nd ed., Oxford Univ. Press, Oxford, 1939.

Department of Mathematics, University of South Florida, Tampa, Florida 33620 